\title{
ASYMMETRIES IN LIMB DARKENING REANALYZED
}

\author{
G. H. ELSTE \\ The Lniversity of Michigan, Department of Astronomy. Ann Arbor, MI 48109-109. L.S.A.
}

(Received 24 August, 1989; in revised form 3 October, 1989)

\begin{abstract}
The cause of the asymmetries in limb darkening reported by Neckel and Labs (1987) is discussed on the basis of new, stray-light-free observations, and found to be of instrumental origin.
\end{abstract}

\section{Introduction}

Neckel and Labs (1987) reported systematic variations in their 1981 limb darkening observations, which indicate perhaps the presence of waves (Neckel and Labs, 1988). Foukal (1989) and Neckel and Labs (1989) debated various possible instrumental explanations.

Early observers used special techniques to avoid and/or correct asymmetries in drift curves caused by the finite time constant of the receiver-recorder system. The best documented work is perhaps that by Pierce et al. (1950). With the improvement of the technology investigators became less concerned. The most surprising work is that of Wittmann $(1978,1980)$, who used the fast scanning technique in search for differences between equatorial and polar limb profiles. He found striking asymmetries in both, E-W and $\mathrm{N}-\mathrm{S}$ scans, the preceding limb being always fainter than the following. No attempts were made to search for the cause, except by checking the mechanical drive. These asymmetries are very probably caused by the photomultiplier hysteresis, which is discussed in detail by Young (1974). The problem was given proper attention in recent limb darkening observations by Rosen et al. (1982), Petro et al. (1984), and Neckel and Labs (1989).

In the following we report about new limb-darkening observations obtained with a coronagraph. These do not show evidence of systematic differences between the two halves of a scan across the disk as found by Neckel and Labs (1987). A careful study of the repetition of their observed features with time leads to a possible explanation.

\section{Observations}

The new observations are carried out with a coronagraph to mainly suppress telescopic stray light. The correction for stray light in most solar telescopes is relatively uncertain. In this case the instrument is the coronagraph of the John Evans Solar Facility at the National Solar Observatory at Sacramento Peak. A small hole of 32 arc sec diameter at the prime focus $(f=8 \mathrm{~m})$ practically eliminates telescopic stray light. Through a Coudé system the coronagraph feeds the $16 \mathrm{~m}$ Littrow spectrograph with entrance slit dimensions of $1 \times 28$ arc sec.

In order to minimize the effects of photomultiplier hysteresis and of the moment of 
inertia of the telescope, it is practical to use the slow scanning technique ( $290 \mathrm{~s}$ from limb to limb) rather than the drift curve technique. Data are obtained for steps of 1 arc sec in $0.15 \mathrm{~s}$. The scanning is achieved by the computer controlled motion of the telescope guiding lens.

We use the image rotation, produced by the Coudé system, to scan in various fixed directions during the day, keeping the slit aligned parallel with the limb. A centering device and program provides for a precise disk center location before the start of each scan. A scan is carried out in four passes: No. 1 from the center to the NW limb for example, and 200 arc sec beyond; No. 2 goes back to the center, and continues without interruption as No. 3 to the SE limb and 200 arc sec beyond; finally No. 4 moves back to disk center. There the centering is checked, and scans with poor centering are discarded. The data sets also contain various other measurements like dark level, scattered light in the spectrograph, and disk center intensities without telescope motion.

In the scanning technique the extinction by the atmosphere constantly changes, firstly because of the change in zenith distance of disk center, and secondly because of the differential zenith distance of the scanning aperture with respect to disk center. In order to take into account the first, we simultaneously record the brightness of the entire disk and its immediate surrounding. A $100 \AA$ bandpass interference filter, centered on the chosen continuum wavelength, eliminates the remaining wavelengths. The second variation is treated in the reduction program.

Two wavelengths were chosen to search for the possible variation of the limb darkening with time in the solar cycle. These are $4451.2 \AA$, already chosen by Petro et al. (1984), and $5011.5 \AA$, for which Wittmann $(1978,1980)$ found systematic asymmetries between opposite limbs. His observations were obtained with the Gregory-Coudé type telescope at Locarno, especially designed for low stray light. His results differ from those by Pierce and Slaughter (1977), obtained with the McMath telescope at Kitt Peak.

\section{Reductions}

In the reduction process we evaluate the change in the transparency for the varying differential zenith distance of the scanning aperture, and apply a proper correction. This depends on the angle between the direction of the scan and the zenith. How big can that correction become? Typical zenith transmission for $4451 \AA$ is 0.82 . For a zenith distance of $60 \mathrm{deg}$ this gives a differential transmission between limb and center of the order of $0.2 \%$. However, the order of scan directions is arranged in such a way to achieve the least change in zenith distance of the scan aperture during the limb to limb scan, pass 2 and 3. As an example, for a rising Sun this scan direction is downward. Thus for most of our observations the correction for varying transmission is considerably smaller than the $0.5 \%$ needed for telescopic stray light in the McMath telescope (Petro et al., 1984). It is important to remember that the correction for differential transmission is straight forward, which is not the case for the instrumental stray light.

The next important step is the precise determination of the location of the Sun's limb 
on data sets, which are considerably affected by seeing. For each of the four passes the location of the limb is determined by the integral method, which is quite independent of the blurring by seeing. In the future we will use the Finite Fourier Transform Definition introduced by Hill, Stebbins, and Oleson (1975). A comparison of the two methods can be found in their paper.

Before further processing it is desirable to eliminate data corresponding to spots, pores, and plage areas. This is accomplished by assigning weights to points, which deviate substantially from the general limb-darkening law. For this we accept the 5 th-order polynominal in $\cos \theta$ derived by Petro et al. (1984). Data in each of the four passes are multiplied by a normalization factor to match this general limb-darkening law. The residuals from that fit are now checked to assign zero weights to all data points for which the absolute value of the residual is larger than $3 \%$. We then display four point averages of the residuals in a graph to provide an opportunity to eliminate poor scans. A sample graph is shown in Figure 1. Disk center is located on the left edge. We disregard points closer than $19^{\prime \prime}$ from the limb. At the lower part of the graph we display the residuals of the simultaneously recorded transparency departing from a linear relation during the four passes. This permits to check if spurious residuals, shown in the upper portion of the graph, can be explained by transparency fluctuations.

The assumption of a linear behavior of the transparency during the $11 \mathrm{~min}$ of the four passes needs some explanation. It was originally planned to always use the directly recorded brightness of the entire disk and its immediate surrounding as a measure for the transparency at disk center. However, several problems arose. For most of the observations in 1986 and 1987 there was no provision for covering the transparency monitor to provide the zero level of that signal. More problematic is the frequently experienced amount of noise. This signal is not digitized near the receiver.

Thus, it became necessary to replace the time variation of the brightness signal of the disk by two linear relations derived from the scan when the telescope points near disk center, i.e., at the beginning of a scan, in the middle, and again at the end of it. The disk brightness actually measured by the transparency monitor is still used for checking purposes in the graph shown in Figure 1.

The further reduction procedure is not important for the remaining part of this contribution, and will be published elsewhere.

\section{Treatment of Residuals}

Most of our limb-darkening measurements were taken at the continuum window at $4451 \AA$. This window is not contained in the extended observations by Neckel and Labs (1987). However, the systematic asymmetries, which these authors found, are quite independent of wavelength, and also present in their $20 \AA$ wide bands as well as in the continuum. In Figures $7(\mathrm{a}-\mathrm{d})$ of their paper they show the difference between limbdarkening polynomials fitted to the west and the east part of drift curves.

For a comparison we wish to make our procedure as similar as possible to theirs. So we will only use the uninterrupted part of our scans from limb to limb, i.e., passes 2 

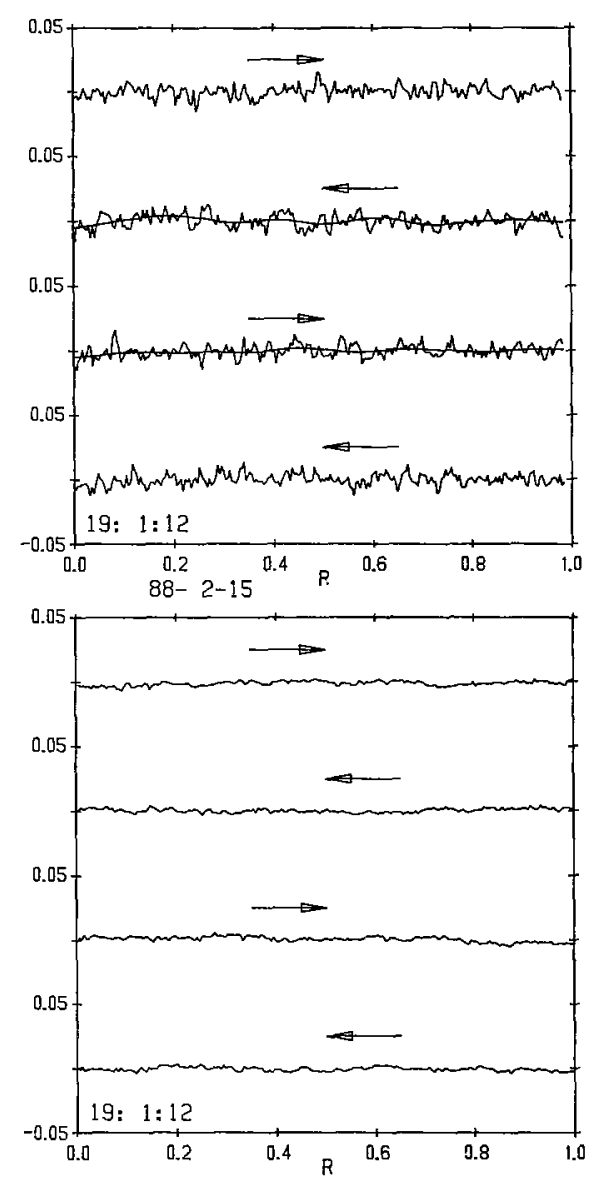

Fig. 1. Residuals of the four passes between disk center and limb relative to the polynomial expression by Petro et al. (1984) for a scan of February 15, 1988, at 19:01 UT, and corresponding residuals from a linear relation of the simultaneously recorded transparency averaged over the disk. Disk center is at the left edge. Arrows mark the direction of the scan.

and 3. Notice that for all passes the residuals are relative to the same limb-darkening polynomial of Petro et al. (1984), using their coefficients. These residuals are then smoothed by means of Fourier transform and filtering of the higher frequencies. The smoothed curves are also drawn in Figure 1 for passes 2 and 3.

The difference in the smoothed residuals of pass 3 minus those of pass 2 are finally displayed in Figure 2. In order to demonstrate the behavior during a day we display them for 11 scans observed on February 15, 1988. These curves do not precisely correspond to the differences shown in the figures of Neckel and Labs (1987). They determined separate polynomials to each half of a drift curve, and then took the difference between the two polynomials. We consider the difference of smoothed residuals relative to the same polynomial to be a better indicator for a wave pattern.

As could be expected our difference curves do show some wavy departures from zero. But there is no indication of a systematic behavior as seen in the observations by Neckel 


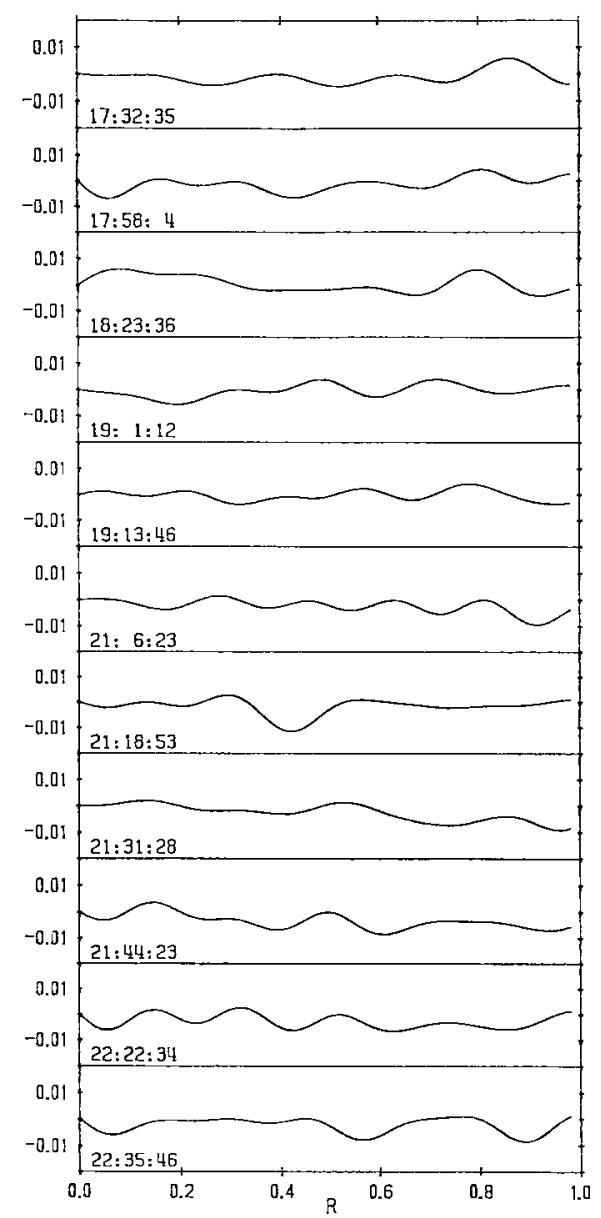

Fig. 2. Differences between the smoothed passes 2 and 3 for 11 scans of February 15, 1988. Disk center is at the left edge.

and Labs (1987), particularly near the limb. Our observed variation of the difference of the smoothed residuals between following and preceding half of a scan is certainly of solar origin.

But let us now apply exactly the same procedure as Neckel and Labs (1987) by determining separate polynomials to the two halfs of a scan. The difference between the polynomials is shown in Figure 3 for the same observing sets as used for Figure 2. We notice the much more extended flat parts, but also the sudden upward or downward bends near the limb, which will be discussed later. Our curves look definitely quite different from those by Neckel and Labs (1987). Let us, therefore, examine their findings more closely. 


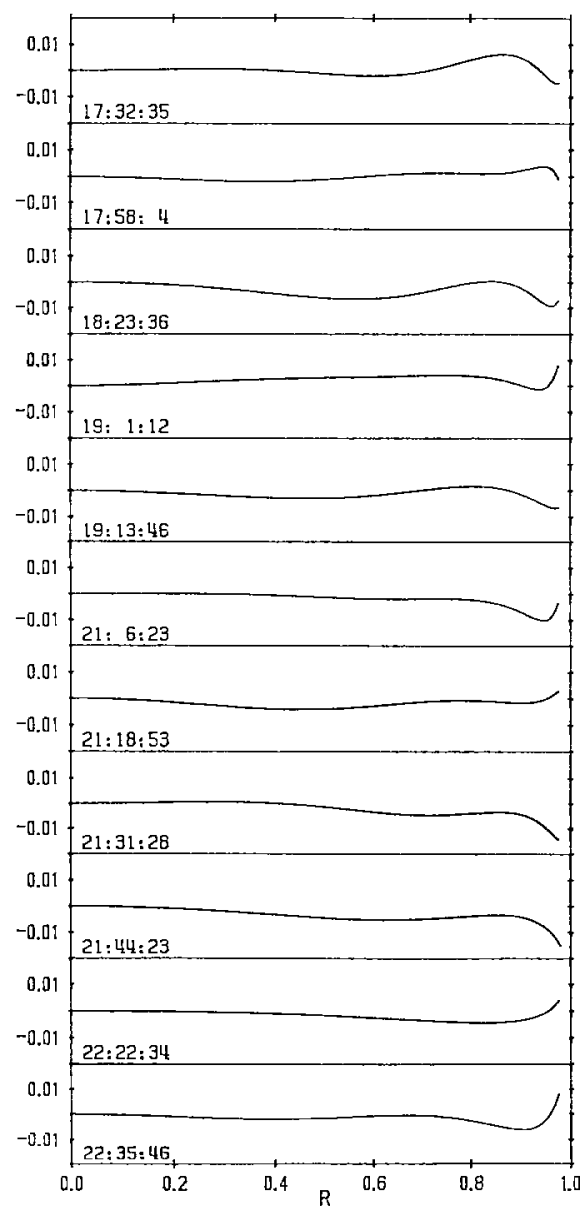

Fig. 3. Differences between 5 th-order polynomials fitted to passes 2 and 3 for the same 11 scans shown in Figure 2.

\section{Discussion of the Systematic Differences between W- and E-Limbs Reported by Neckel and Labs}

The difference between limb-darkening polynomials fitted to the two halves of drift curves is shown in their Figure 7(a) for two continuum windows. This difference slowly increases from disk center to about $R=0.4$ and then decreases to a minimum at about $R=0.7$, where $R$ is in units of the solar radius. Then there is a more pronounced maximum just before $R=0.9$. Such a behavior is observed at several days, and at different times: on 30.4. at 7:24 and 7:31 UT, as well as on 29.4 at 18:02 and 18:07 UT. The limb darkening observed for a $20 \AA$ wide spectral band $6559 \AA$ on 24.4 . at 17:09 UT is again of the same kind, Figure 7(b). Other shapes are found for $5410 \AA$ on 23.4. at 6:43 and 22.4. at 16:50 UT, as well as for 3298 in the UV. It is hard to believe that the same pattern should be present on the Sun for many hours and be different on the next day. 
More revealing is the variation in shape of the curves shown in their Figure $8(a-b)$. On the 23.4.81 between $6: 30$ and $8: 00$ UT the maxima occur close to $R=0.95$. On the 24.4.81 between $14: 30$ and 16:00 UT the maxima are located about at $R=0.90$. On the 29.4.81 between $9: 30$ and 10:30 UT there are minima close to $R=0.90$. However, we also notice some cases on the 24.4 .81 in the morning when occasionally two successive drift curves at the same wavelength are really different. But these are exceptions.

The striking similarity of most curves of a single day regardless of wavelength, together with differences between sets of curves of different days just tells us that something has changed from one day to another. If we ask what in the instrument could possibly be different, we come to the conclusion that this can only be the collimation of spectrograph and telescope. From my experience with the McMatch tower and the main spectrograph line-up I remember the very delicate procedure every morning using the engineering transit. An asymmetrical illumination of the parabolic telescope mirror by the elliptical beam from the siderostat stays fixed with respect to the E-W direction during the day.

Rosen et al. (1982), who also used the McMath tower at Kitt Peak, compare in their Figure 1 the difference in the average of limb darkening observations obtained on two consecutive days at the same wavelength. This difference shows a similar systematic behavior close to the limb, $\cos \theta=0.37$, as found by Neckel and Labs (1987).

But how can a slightly different collimation lead to systematic asymmetries in the limb darkening? Of course we only deal with effects in the one percent range. It is simply the relatively large amount of telescopic scattered light together with asymmetrical vignetting in the telescope which must be the cause. The larger asymmetries at shorter wavelength, where the stray light is stronger could only in part support that interpretation since solar structure variations are also known to increase with decreasing wavelength.

\section{Limb Intensity Profile and the Polynomial Representation}

We finally have to look for the origin of the sudden turns near the limb of Figure 3 . For $0.06<\cos \theta<0.2$ the limb intensity distribution on a logarithmic scale appears to be almost linear. Figure 4 displays three limb profiles obtained with high spatial resolution. Results of Gaustadt and Rogerson (1961) from project Stratoscope for $540 \mathrm{~nm}$ are shown as squares. From the results of Ballario and Godoli (1955) for many wavelengths obtained during the 1954 eclipse those for $450 \mathrm{~nm}$ are displayed as triangles. For clarity they are shifted downwards by 0.1 dex, which does not affect the general behavior of the limb profile. Neckel (1958) obtained for the same wavelength intensities for only a few points shown as $N$. Very similar limb profiles result from many other investigations as discussed in detail by Mädlow (1961) who also describes the various techniques.

In contrast to the smooth limb profiles from single shots corrected for seeing, our slow scans are strongly affected by noise near the limb due to seeing. From our observations of February 15, 1988 we select the scan of 19:01 UT. This does not show much 


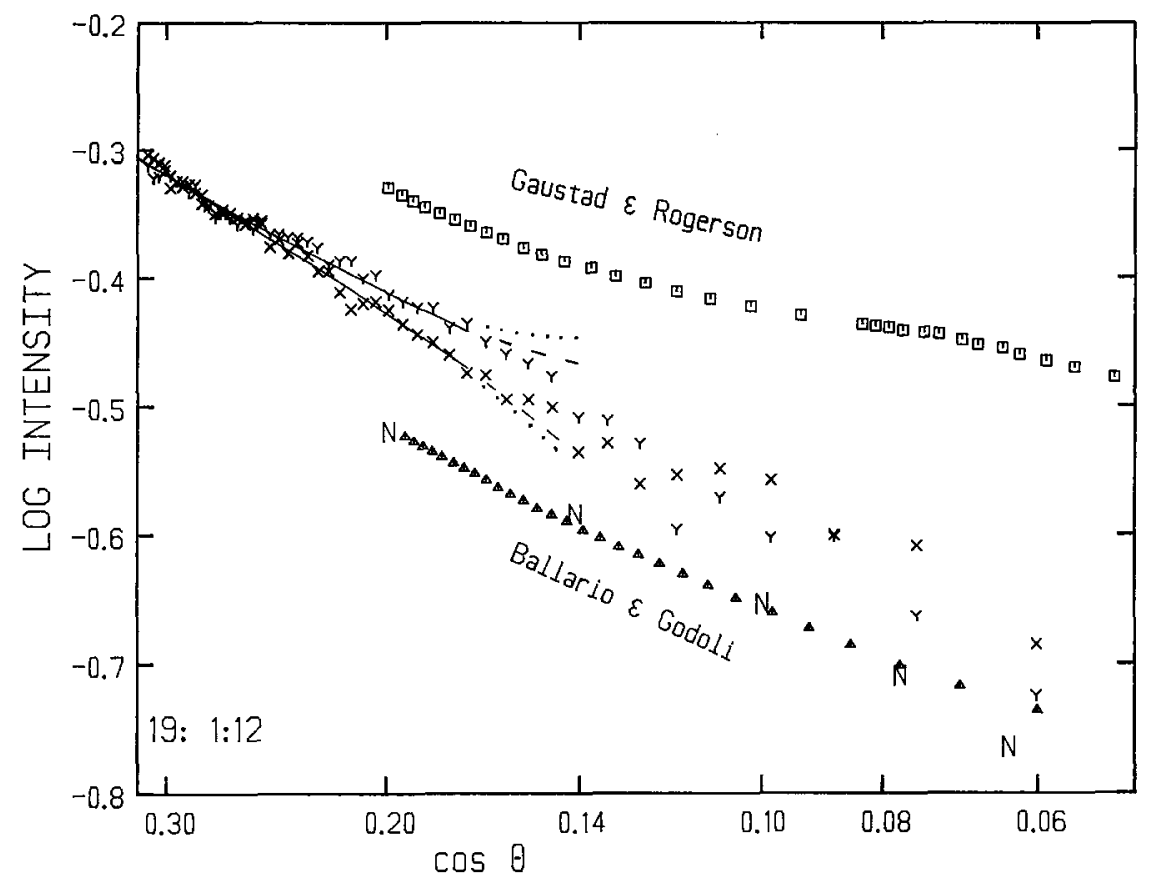

Fig. 4. High-resolution intensity profiles of the extreme limb and a sample of slow scan observations, marked $X$ and $Y$, with their polynomial representation (solid curves) and extrapolation (broken curves) as discussed in the text.

difference between the two limbs as seen in Figure 2. But the difference between the two separate fifth-order polynomials diverges near the two limbs, see Figure 3. In Figure 4 the points from the limb to center pass are marked as $X$, those from the center to limb pass by $Y$. The increasing influence of the seeing with approach of the limb is obvious. Near $\cos \theta=0.2$ the points of the outward scan are located 0.03 dex above those of the inward scan. This may well be due to local differences and/or seeing. The run of the fifth order polynomials for the two passes is shown as solid curves within the region of fit. One realizes that the cause for their divergence is the just mentioned difference near the boundary of fit. In order to make it more obvious we indicate the run beyond the boundary by broken lines.

The expectation that polynomials in powers of $\ln (\cos \theta)$ would behave more reasonable near the limb than those in powers of $\cos \theta$ however did not substantiate. When we fit polynomials in $\ln (\cos \theta)$ to the data in the two passes 2 and 3 of our observations we obtain the dotted curves in Figure 4.

We must conclude that the sudden divergence of polynomial representations near the limb is due to the different scatter of the observations neart the boundary of fit at the two opposite limbs. When Neckel and Labs find that behavior repeatedly in one day's drift curves, one cannot avoid the conclusion that there must be a systematic trend in the observations near the limb. 


\section{Conclusion}

We have learned that the systematic differences of 5 th-order polynomials found by Neckel and Labs (1987) are produced by slight differences in the collimation of spectrograph and telescope, resulting in different stray light distribution. The description of differences between two halves of a drift curve or scan is more realistically done by differences in residuals relative to the same analytic profile. High-order polynomials may lead to unrealistic description of the intensity distribution near the limb.

\section{References}

Ballario, M. C. and Godoli, G.: 1955, Mem. Obs. Astrophys. Arcetri 70, 93.

Foukal, P.: 1989, Solar Phys. 120, 249.

Gaustadt, J. E. and Rogerson, J. B.: 1961, Astrophys. J. 134, 323.

Hill, H. A., Stebbins, T. T., and Oleson, J. R.: 1975, Astrophys. J. 200, 484.

Mädlow, M.: 1961, Astron. Nachr. 286, 271.

Mitchell, W. E., Jr.: 1982, Solar Phys. 80, 3.

Neckel, H.: 1958, Z. Astrophys. 44, 153.

Neckel, H. and Labs, D.: 1987, Solar Phys. 110, 139.

Neckel, H. and Labs, D.: 1988, in P. Foukal (ed.), Solar Radiative Output Variations, p. 201.

Neckel, H. and Labs, D.: 1989, Solar Phys. 120, 205.

Petro, L. D., Foukal, P. V., Rosen, W. A., Kurucz, R. L., and Pierce, A. K.: 1984, Astrophys. J. $283,426$.

Pierce, A. K. and Slaughter, C. D.: 1977, Solar Phys. 51, 25.

Pierce, A. K., McMath, R. R., Goldberg, L., and Mohler, O. C.: 1950, Astrophys. J. 112, 289.

Rosen, W. and Chromey, F.: 1985, Astron. J. 90, 139.

Rosen, W. A., Foukal, P. V., Kurucz, R. L., and Pierce, A. K.: 1982, Astrophys. J. 253, L89.

Wittmann, A.: 1978, Astron. Astrophys. 64, 91.

Wittmann, A.: 1980, Astron. Astrophys. 83, 312.

Young, A. T.: 1974, in N. Carlton (ed.), Methods of Experimental Physics, Vol. 12, Part A, Academic Press, New York, p. 1. 